\title{
Inference of the microwave absorption coefficient from stray radiation measurements in Wendelstein $7-\mathrm{X}$
}

\author{
D Moseev ${ }^{1}$, H P Laqua ${ }^{1}$, S Marsen ${ }^{1}$, T Stange ${ }^{1}$, H Braune ${ }^{1}$, \\ F Gellert ${ }^{1,2}$, J W Oosterbeek ${ }^{3}$ and the Wendelstein 7-X team \\ ${ }^{1}$ Max-Planck-Institut für Plasmaphysik, Greifswald, Germany \\ ${ }^{2}$ Ernst-Moritz-Arndt-Universität Greifswald, Greifswald, Germany \\ ${ }^{3}$ Eindhoven University of Technology, Eindhoven, The Netherlands \\ E-mail: dmitry.moseev@ipp.mpg.de
}

\begin{abstract}
.
The efficiency of electron cyclotron heating is determined by the microwave absorption of the plasma. Good microwave absorption is also crucial for the machine safety. In this paper we present a method of evaluating the microwave absorption coefficient from stray radiation measurements. Evolution of the X2 microwave absorption coefficient in Wendelstein 7-X during the start up phase is presented in this paper, as well as an estimate of the $\mathrm{O} 2$ absorption coefficient.
\end{abstract}

15 April 2016

Electron cyclotron resonance heating $(\mathrm{ECRH})$ is the main heating mechanism in Wendelstein 7-X [1-3]. The ECRH system is capable of delivering up to $5 \mathrm{MW}$ at 140 $\mathrm{GHz}$ in the first operational phase OP1.1 and up to $9 \mathrm{MW}$ in the next campaign OP1.2 scheduled for 2017. Operating at such high power, the knowledge of the microwave absorption is crucial for understanding the physics of heating and for the machine safety. Wendelstein 7-X is equipped with five absolutely calibrated sniffer probes, equally toroidally distributed in the machine [4]. Sniffer probes measure the distribution of ECRH stray radiation energy flux in the machine. The distribution of stray radiation is determined by power balance and described by the multiresonator model [5]:

$$
P_{k}^{\text {in,stray }}=A_{e f f, k} \cdot p_{k}+\sum_{j \neq k} S_{j, k}\left(p_{k}-p_{j}\right),
$$

where $P_{k}^{\text {in,stray }}$ is input power of stray radiation in the resonator $k ; A_{e f f, k}$ is an effective black surface area of the resonator $k$, that absorbs all the incident stray radiation. It can be expressed as in Eq. (2); $p_{k}$ is the stray radiation energy flux in resonator $k$; index $j$ runs through all the resonators in the considered system except resonator $k ; S_{j, k}$ is an interface area between resonator $j$ and resonator $k$. 
Effective absorption area is defined in the following way:

$$
A_{\text {eff }}=\sum_{i=1}^{M} A_{i} \eta_{i}
$$

where index $i$ runs through the different absorption areas of the considered cavity with the surface area $A_{i}$ and microwave absorption $\eta_{i}$. In fact, the value of $A_{\text {eff }}$ determines the quality factor of a resonator: the bigger the effective absorption area is, the smaller the $\mathrm{Q}$ factor of the resonator.

The input power for stray radiation in application to Wendelstein 7-X can be expressed as a function of gyrotron power and directed beam absorption:

$$
P_{k}^{\text {in,stray }}=P_{k}^{\text {gyrotron }}\left(1-\eta_{\text {dir.beam }}\right),
$$

where $P_{k}^{\text {in,stray }}$ is the input power of stray radiation in resonator $k$ and a source term in Eq. (1); $P_{k}^{\text {gyrotron }}$ is the gyrotron power injected into the resonator $k ; \eta_{\text {dir.beam }}$ is an absorption coefficient of the directed beam. One should note that $\eta_{\text {dir.beam }}$ is not the single pass absorption of the ECRH beam by the plasma, although it is a related quantity. Assuming that all the gyrotron radiation is either absorbed by plasma or goes into stray radiation, $\eta_{\text {dir.beam }}$ shows which part of the gyrotron power is absorbed by the plasma. It is illustrated in Fig. 1. An important property of directed beam absorption is that it cannot be smaller than single pass absorption.

The microwave absorption coefficient of the plasma $\eta_{\text {dir.beam }}$ can be found by forward modeling, when the output of the multiresonator model is compared with the sniffer probe readings. The ECRH launchers are located in modules one and five of Wendelstein 7-X. Due to the relatively large influence of the directed beam on the sniffer probe measurements in these modules, only the measurements of sniffer probes from modules two, three, and four are used for the fitting. An example of applying the method is illustrated in Fig. 2. The figure shows an evolution of the microwave absorption coefficient for X2 polarized ECRH beam. Withing $10 \mathrm{~ms}$ the absorption coefficient increases from about $25 \%$ to nearly $100 \%$. The total injected RF power is displayed in the middle panel. The change in the energy flux of stray radiation in module three, the most remote position in the vessel from the ECRH launchers, is depicted in the lower panel of the graph. The measurement was done by the sniffer probe diagnostic with 0.5 ms averaging. Correct estimation of the distribution of stray radiation sources is not obvious to figure out since there is no clear transition between the directed reflection of the ECRH beam and stray radiation. This directly affects quality of the fits presented further in this paper. In the presented analysis, we consider that the stray radiation originates from the same module of the stellarator where the ECRH launcher is located.

In order to demonstrate the sensitivity, the method is tested with the modulated gyrotron power, launched either in X2 or in $\mathrm{O} 2$ mode. Only one gyrotron is modulated while another five are used to sustain the plasma. In the first experiment (27.01.2016, 15:17:34 UTC) the bulk plasma was heated by $3.5 \mathrm{MW}$ ECRH in X2 mode. The resulting plasma has an electron temperature of $8 \mathrm{keV}$ and density of $3 \cdot 10^{19} \mathrm{~m}^{-3}$. The power of the D5 gyrotron installed in module 5 is modulated in the range from 120 

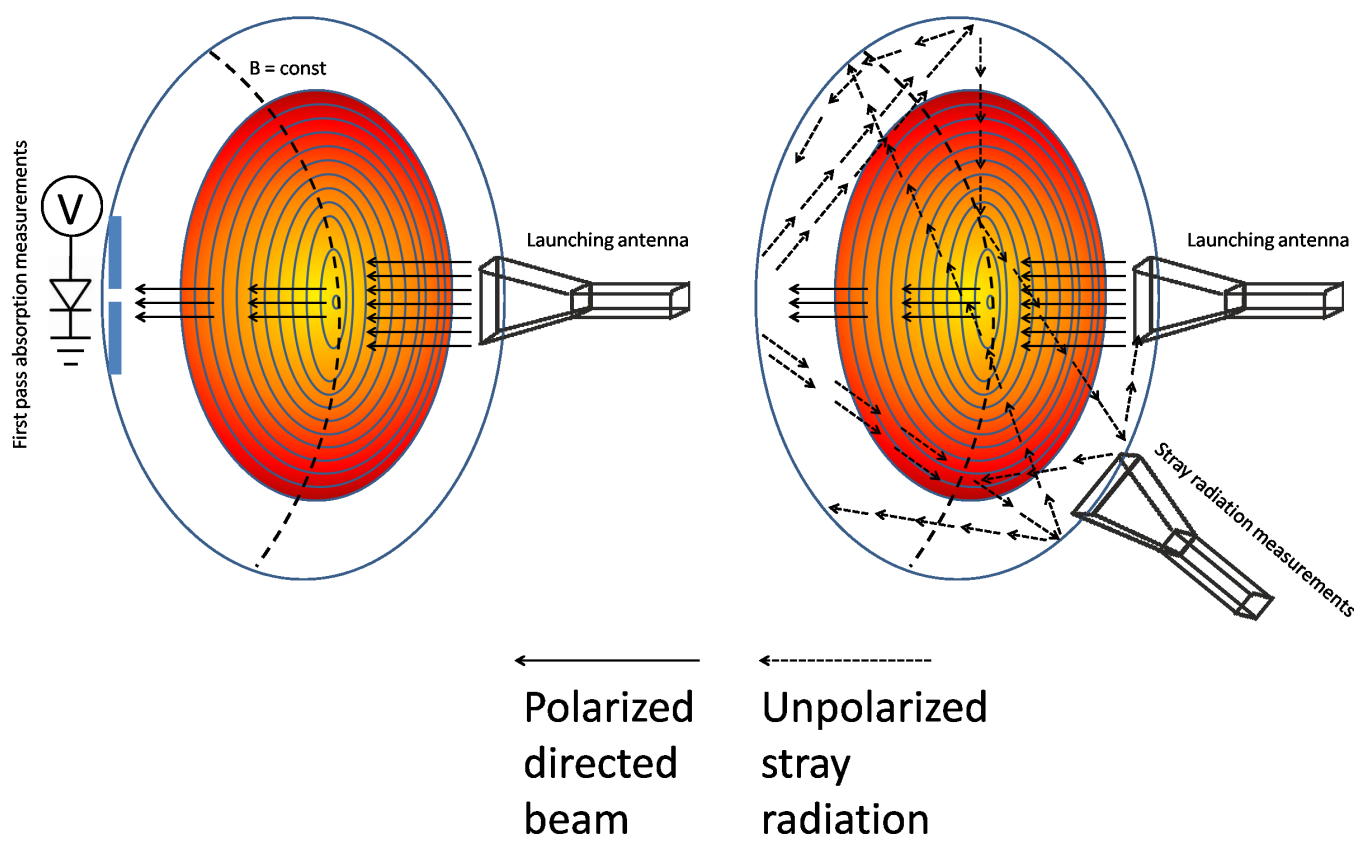

Figure 1. Sketch illustrating the difference between first pass absorption in plasma (left) and the directed beam absorption. The directed polarized radiation is depicted by solid arrows and randomly polarized isotropic stray radiation is represented by dashed lines (right). The first pass absorption describes the attenuation of directed and polarized radiation after passing through plasma and the directed beam absorption coefficient describes which part of the input power from the gyrotron is not converted into stray radiation.

$\mathrm{kW}$ to $200 \mathrm{~kW}$ in $\mathrm{X} 2$ mode. The power of the D5 gyrotron is shown in the upper left panel of Fig. 3. In order to investigate the effect of this particular source on the stray radiation, conditional averaging of the sniffer probe signals is applied and the averaging is conditional to the gyrotron modulation phase. Red and black markers denote time stamps of the maximum and minimum power during the modulation. The contribution of the D5 gyrotron to the total RF power is displayed in the upper right panel of the same graph. The red and black markers depict the same time as in the upper left panel. The corresponding energy flux of stray radiation, measured by the sniffer probe in module three, is shown in the bottom left panel. It is difficult to distinguish between the red and the black markers, apparently the power modulation brings little difference to the total stray radiation pattern. Indeed, the results of conditional averaging for the sniffer probes in modules two, three, and four, shown as blue bars in the bottom right panel of Fig. 3, demonstrate very low values. The fit of the data, demonstrated in the same panel by red bars, results in the microwave absorption coefficient for the X2 mode in such plasma of $96.5 \%$.

The next experiment on 27.01.2016 at 15:22:59 is a repeat of the previous with the same plasma parameters, however the D5 gyrotron is operating in the O2 mode. The corresponding time trace of stray radiation in module three is shown in the left panel of Fig. 4. The change in stray radiation level due to the power modulation of the D5 

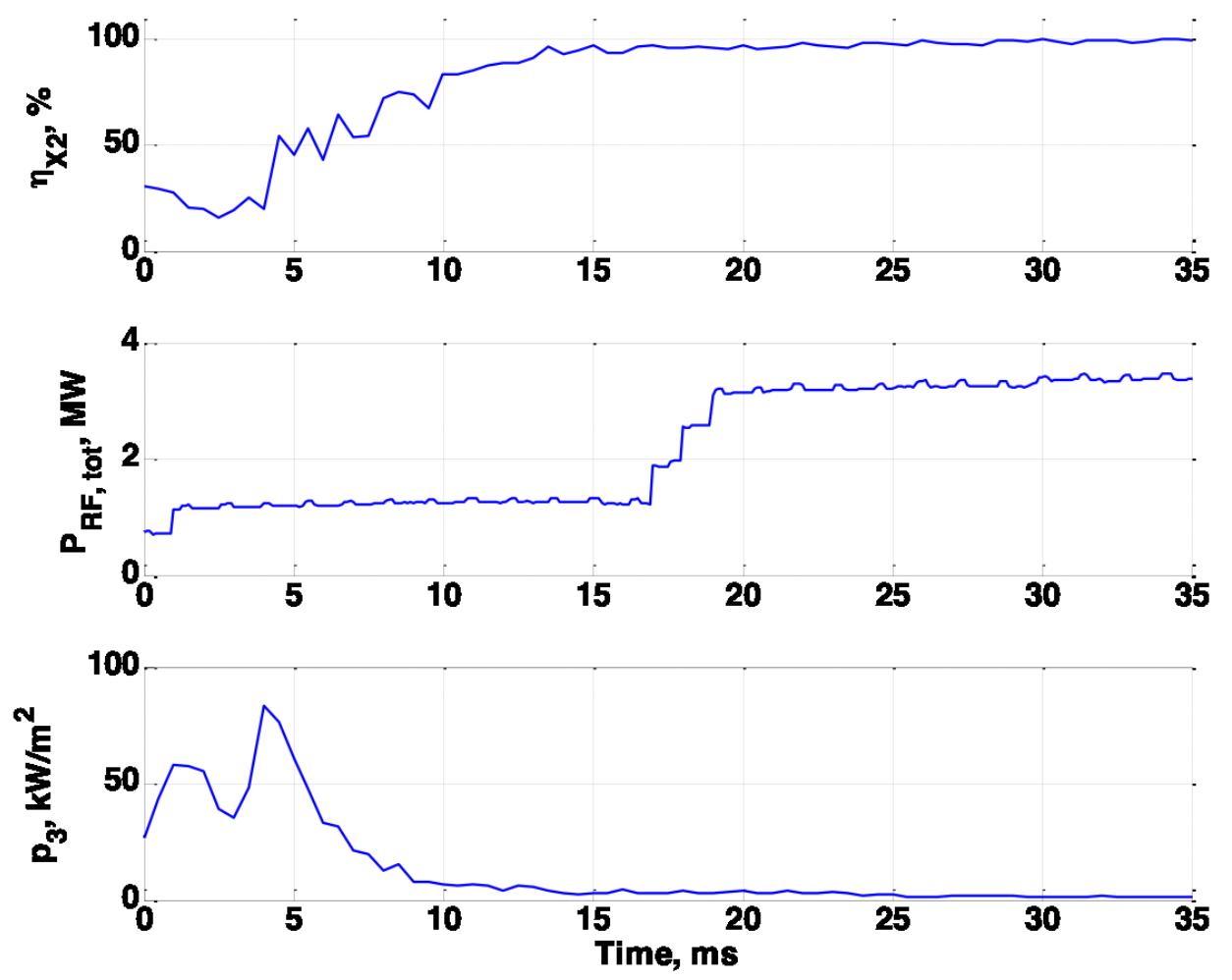

Figure 2. Plasma start-up phase of the Wendelstein 7-X experiment with helium plasmas on 27.01.2016 at 15:11:46 UTC. ECRH switches on at $0 \mathrm{~ms}$. Inferred evolution of the microwave absorption coefficient for the $\mathrm{X} 2$ polarized ECRH radiation is shown in the upper panel; total ECRH injected into Wendelstein 7-X is depicted in the middle panel; stray radiation energy flux in module three, measured by the sniffer probe diagnostic is shown in the lower panel.

gyrotron obtained by conditional averaging and its numerical fit are depicted in the right panel of Fig. 4. The microwave absorption coefficient for $\mathrm{O} 2$ mode in such plasma equals to $76 \%$, which agrees well with TRAVIS [6] simulations for first path absorption of $\mathrm{O} 2$ of $72 \%$ for such plasma parameters. The average standard deviation of conditional averaging of the three sniffer probes is $19 \%$. The performed sensitivity analysis shows, that with the given error-bars on the sniffer probe reading, the microwave absorption coefficient of the plasma varies between $68 \%$ to $79 \%$.

\section{Conclusions}

A new method for deducing the microwave absorption coefficient of plasma is developed. The method is based on forward modeling of the stray radiation distribution in the vacuum vessel of the machine with plasma and find fitting values for the effective microwave absorption area and the microwave absorption coefficient. The microwave absorption coefficient found in such a way represents the ratio of power that has never 

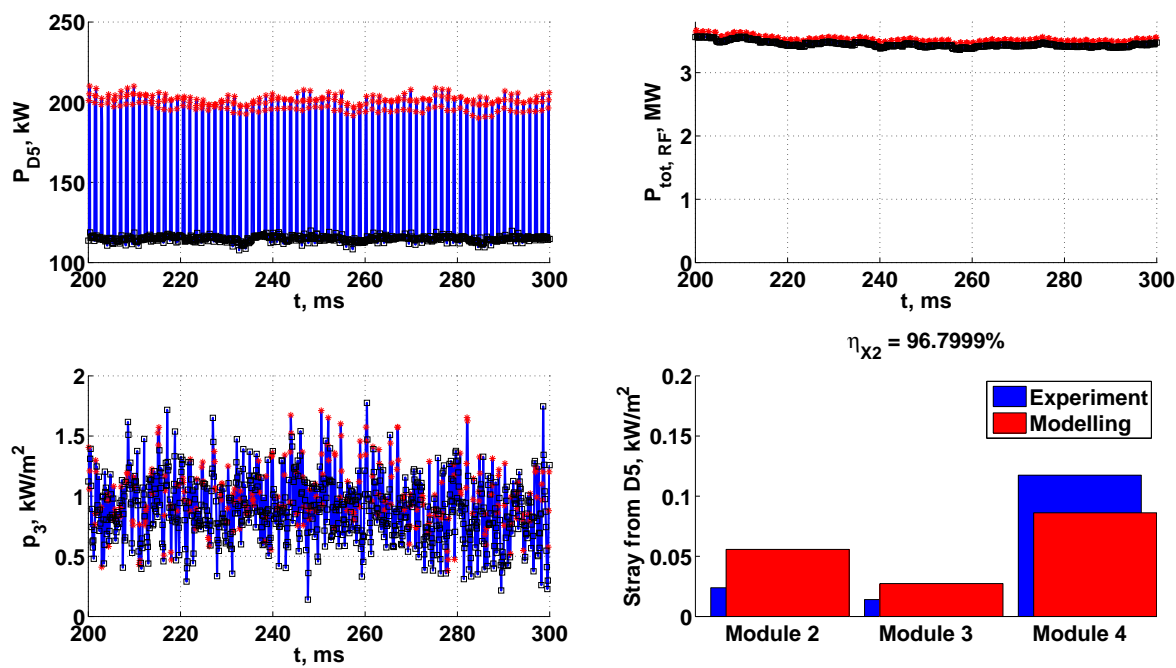

Figure 3. Wendelstein 7-X experiment on 27.01.2016 at 15:17:34 UTC. Upper left panel: power modulation of the D5 gyrotron in X2 mode, red and black markers indicate the time of maximum and minimum power during modulation. Upper right panel: total RF power injected into the plasma. Bottom left panel: stray radiation energy flux in module three. Bottom right panel: blue bars represent the change in stray radiation energy flux due to the D5 gyrotron power modulation obtained by conditional averaging, red bars depict numerical fit to the data.

contributed to the stray radiation level in the machine and the injected microwave power. It is strongly related to the first pass absorption coefficient and by that we mean that the first pass absorption coefficient cannot exceed the microwave absorption coefficient computed by the presented method. However, in devices with highly reflective walls like Wendelstein 7-X during the first operation phase, the two coefficients are rather close to each other. This statement is supported by ray-tracing using the TRAVIS code.

It is also worth noticing that in its first experimental campaign Wendelstein 7-X proved that it can provide sufficient absorption of $\mathrm{O} 2$, indicating that the high density operation with O2 EC heating should be possible.

\section{Acknowledgments}

The authors express their gratitude to Prof. Robert Wolf for fruitful discussion and proof reading. This work has been carried out within the framework of the EUROfusion Consortium and has received funding from the Euratom research and training programme 2014-2018 under grant agreement No 633053. The views and opinions expressed herein do not necessarily reflect those of the European Commission. 

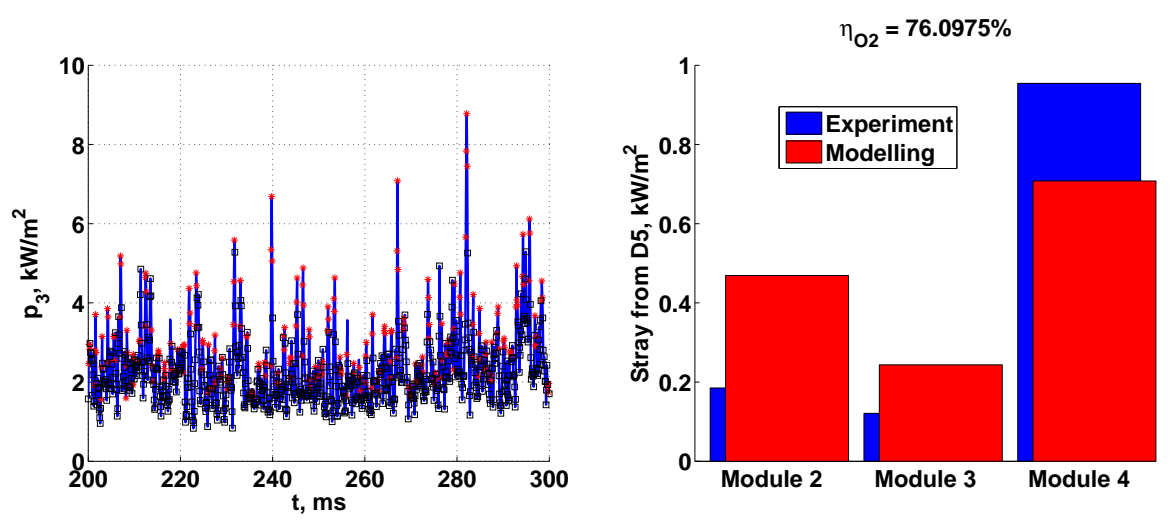

Figure 4. Wendelstein 7-X experiment on 27.01.2016 at 15:22:59 UTC. Left panel: stray radiation energy flux in module three. Red and black markers show the values of the stray radiation at maximum and minimum power of the D5 gyrotron during power modulation. Right panel: blue bars represent the change in stray radiation energy flux due to the D5 gyrotron power modulation obtained by conditional averaging, red bars depict the numerical fit to the data.

\section{References}

[1] V. Erckmann, P. Brand, H. Braune, G. Dammertz, G. Gantenbein, W. Kasparek, H. P. Laqua, H. Maassberg, N. B. Marushchenko, G. Michel, M. Thumm, Y. Turkin, M. Weissgerber, and A. Weller. Electron Cyclotron Heating for W7-X: Physics and Technology. Fusion Science and Technology, 52(2):291-312, August 2007.

[2] V. Erckmann, P. Brand, H. Braune, G. Dammertz, G. Gantenbein, W. Kasparek, H. P. Laqua, G. Michel, M. Thumm, and M. Weissgerber. The $140 \mathrm{GHz}, 10 \mathrm{MW}$, CW ECRH Plant for W7-X: A Training Field for ITER. In Fusion Energy 2006, pages IT/2-4Rd, Chengdu, 2007. International Atomic Energy Agency.

[3] J Geiger, R C Wolf, C Beidler, A Cardella, E Chlechowitz, V Erckmann, G Gantenbein, D Hathiramani, M Hirsch, W Kasparek, J Kiß linger, R König, P Kornejew, H P Laqua, C Lechte, J Lore, A Lumsdaine, H Maaß berg, N B Marushchenko, G Michel, M Otte, A Peacock, T Sunn Pedersen, M Thumm, Y Turkin, A Werner, and D Zhang. Aspects of steady-state operation of the Wendelstein 7-X stellarator. Plasma Physics and Controlled Fusion, 55(1):014006, January 2013.

[4] D Moseev, H P Laqua, S Marsen, T Stange, and H Braune. Absolute calibration of sniffer probes on Wendelstein 7-X. submitted to RSI, 2016.

[5] H P Laqua, V Erckmann, and M Hirsch. Distribution of the ECRH stray radiation in fusion devices. In 28th EPS Conference on Contr. Fusion and Plasma Phys., number June, page P3.099, 2001.

[6] N.B. Marushchenko, Y. Turkin, and H. Maassberg. Ray-tracing code TRAVIS for ECR heating, EC current drive and ECE diagnostic. Computer Physics Communications, 185(1):165-176, January 2014. 